\title{
Variaciones en el número de metámeros del músculo recto abdominal. Una guía para la lipoescultura de alta definición
}

\section{Variation in the number of metamers of rectus abdominis muscle. A high definition lipoesculture guide}

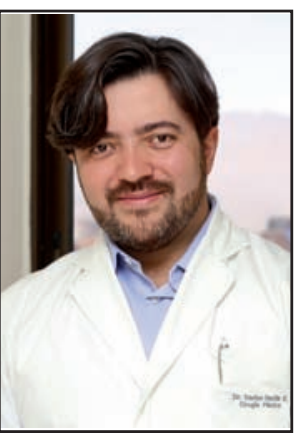

Danilla Enei, S.

Lorena LOPEZ-GARCÍA****, Evelyn LEÓN-CABRERA****, Juan-Pablo CISTERNAS-VERA*,

Introducción y Objetivo. El recto abdominal es un músculo que consta, generalmente, de 3 inserciones tendinosas transversas. La lipoescultura de alta definición apunta a delinear la musculatura abdominal, sin embargo, una de sus dificultades es determinar mediante la anatomía de superficie el número de metámeros presentes.

Nos planteamos describir las variaciones en el número de metámeros del músculo recto del abdomen en la población chilena mediante análisis de tomografía computarizada de abdomen y pelvis, así como determinar si existe un patrón para la marcación quirúrgica de los metámeros.

Material y Métodos. Empleamos una muestra aleatoria de 200 pacientes que cumplían los criterios de inclusión: mayores de 18 años y menores de 65 años, estudio de tomografía computarizada de abdomen y pelvis con secuencia coronal realizado en el Hospital Clínico de la Universidad de Chile entre enero y mayo del 2017; y de exclusión: patología de la pared abdominal, cirugía abdominal previa, estudio imagenológico inadecuado.

Describimos el sexo, número de inserciones tendinosas supra e infraumbilicales, ángulo costal corto, ángulo costal completo, diámetro xifo-umbilical y umbílico-púbico. Analizamos los datos con STATA ${ }^{\circledR}$ v13, considerando un valor estadísticamente significativo de $\mathrm{p}<0.05$ e intervalos de confianza de $95 \%$.

Resultados. Del total de paciente, 100 (50\%) de los pacientes fueron mujeres. La media de edad fue de $40,9 \pm 12.1$ años, y 154 pacientes $(77 \%)$ tenían 3 metámeros. No evidenciamos diferencias significativas entre ambos sexos $(\mathrm{p}=0.393)$. Ni en el análisis de la distancia xifo-umbilical $(\mathrm{p}=0.185)$ ni de la umbílico-púbica $(\mathrm{p}=0.327)$ entre sexos respectivamente. El número de metámeros se encuentra en relación inversa al tamaño de los mismos. La angulación del reborde costal en relación al xifoides es significativamente menor en mujeres $(69.4 \pm 15.2$ frente a $80.2 \pm 14.2)$.

Conclusiones. La anatomía más frecuente del músculo recto del abdomen en la población chilena corresponde a 3 metámeros supraumbilicales por cada recto abdominal. Cuanto menor sea la relación entre la distancia xifoumbilical / distancia xifo-púbica, menor cantidad de metámeros tiene el músculo recto. No fue posible predecir en forma precisa el número de metámeros mediante otra medida antropométrica.

\begin{tabular}{|c|c|c|}
\hline Palabras clave & $\begin{array}{l}\text { Abdom } \\
\text { Liposu } \\
\text { Anatom }\end{array}$ & $\begin{array}{l}\text { n, Músculo recto abdominal, } \\
\text { ción, Lipoescultura, } \\
\text { í regional. }\end{array}$ \\
\hline \multicolumn{3}{|c|}{ Nivel de evidencia científica $\quad 4 b$ Diagnóstico/Terapéutico } \\
\hline \multicolumn{2}{|c|}{$\begin{array}{l}\text { Recibido [esta versión] } \\
\text { Aceptado }\end{array}$} & 21 diciembre/2017 \\
\hline
\end{tabular}

Background and Objective. Rectus abdominis muscle usually have 3 tendinous intersections. The aims of high definition abdominal liposculpture is to delineate the musculature. One of its difficulties is to determine by the surface anatomy the number of metamers presents.

The aim of this study is to describe the variations in the number of metamers of the rectus abdominis muscle in the Chilean population and determine if there is a pattern that helps to the surgical mark of the abdominis metamers.

Methods. Randomized sample of 200 patients who met inclusion criteria: $>18$ years of age and under 65 years, tomographic study of the abdomen and pelvis with coronal sequence, performed at the Clinical Hospital of the University of Chile between January and May 2017; and exclusion: pathology of the abdominal wall, previous abdominal surgery or inadequate imaging study. Sex, number of tendinous intersections supra and infraumbilical, short costal angle, complete costal angle, xipho-umbilical distance and umbilico-pubic measures were described. The data were analyzed with $\mathrm{STATA}^{\oplus} \mathrm{v} 13$, being considered statistically significant with $\mathrm{p}<0.05$ and $95 \%$ confidence intervals.

Results. One hundred patients $(50 \%)$ were women, mean age $40.9 \pm 12.1$ years, and 154 patients $(77 \%)$ had 3 metamers. There were no significant differences by $\operatorname{sex}(\mathrm{p}=0.393)$. Neither in the analysis of the xipho-umbilical distance $(\mathrm{p}=0.185)$ and the umbilical-pubic distance $(\mathrm{p}=0.327)$ by genders. The number of metamers is in inverse relation to its size. The angulation of the costal ridge relative to the xiphoid is significantly lower in women $(69.4$ \pm 15.2 versus $80.2 \pm 14.2$ )

Conclusions. The most common rectus abdominis muscle pattern in the Chilean population corresponds to 3 supra umbilical metamers for each side (six-pack). The smaller the relationship between the xifo-umbilical distance / xifo-pubic distance, less number of metamers of the rectus abdominis. It was not possible to predict the number of metamers by another anthropometric measurement.

\begin{tabular}{|lr|}
\hline Key words & \multicolumn{1}{c|}{$\begin{array}{l}\text { Abdomen, Abdomen rectus muscle, } \\
\text { Liposuction, Liposculpture, } \\
\text { Regional anatomy. }\end{array}$} \\
Level of evidence & 4b Diagnostic/Therapeutic \\
Received lthis version] & 21 december/2017 \\
Accepted & 30 january/2018 \\
\hline
\end{tabular}

Conflicto de intereses: los autores declaran no tener ningún interés financiero relacionado con el contenido de este artículo.

* Cirujano Plástico, Equipo de Cirugía Plástica y Reparadora, Departamento de Cirugía.

** Investigador Postdoctorado en Cirugía Plástica, Departamento de Cirugía.

*** Residente de Cirugía General, Departamento de Cirugía.

**** Tecnólogo Médico, Servicio de Imagenología,

Hospital Clínico de la Universidad de Chile, Santiago, Chile. 


\section{Introducción}

La pared abdominal es la estructura que se encarga de proteger y contener a los órganos abdominales. El recto del abdomen es un músculo que se extiende en la superficie anterior del abdomen, envuelto por una fascia y separado al medio por la presencia de la línea alba. Se inserta por cefálico entre la quinta y séptima costillas y el proceso xifoideo, y a caudal en el pubis. Generalmente consta de 3 inserciones tendinosas transversas: una a nivel del margen costal, otra a nivel umbilical y otra entre ambas, conformando la estructura metamérica del músculo recto abdominal. ${ }^{(1,2)} \mathrm{A}$ veces, hay 1 o 2 inserciones incompletas por debajo del ombligo. ${ }^{(3-5)}$ Coloquialmente se les llama 6-pack y desde tiempos de la Grecia Clásica se considera un signo de atractivo en el abdomen masculino y femenino (Fig. 1). Actualmente, los cánones de belleza han girado hacia cuerpos atléticos tanto en hombres como en mujeres, y el advenimiento de redes sociales como Instagram y Pinterest ha difundido ampliamente este tipo de belleza.

Por el lado de la cirugía, Mentz $1993^{(6)}$ y Hoyos $2007^{(7)}$ publicaron una variación de la técnica tradicional de liposucción a la que llamaron Abdominal etching y High definition liposculptre respectivamente; ambas apuntaban a delinear la musculatura para producir como resultado un cuerpo más atlético y de apariencia musculada (Fig. 2.)

Una de las dificultades que presenta la técnica es determinar la anatomía de superficie del músculo recto abdominal y el número de metámeros o vientres sobre el ombligo, que pueden ser 2, 4, 6 u $8{ }^{(8-10)}$ En pacientes delgados puede ser relativamente sencillo, pero en pacientes con adiposidad en la pared anterior del abdomen se hace dificultoso determinar la anatomía con exactitud.

El objetivo del presente estudio es describir las variaciones en el número de metámeros del músculo recto del abdomen en la población chilena mediante el análisis de tomografías computarizadas de abdomen y pelvis, así como determinar si existe algún patrón que guie al cirujano en la marcación quirúrgica.
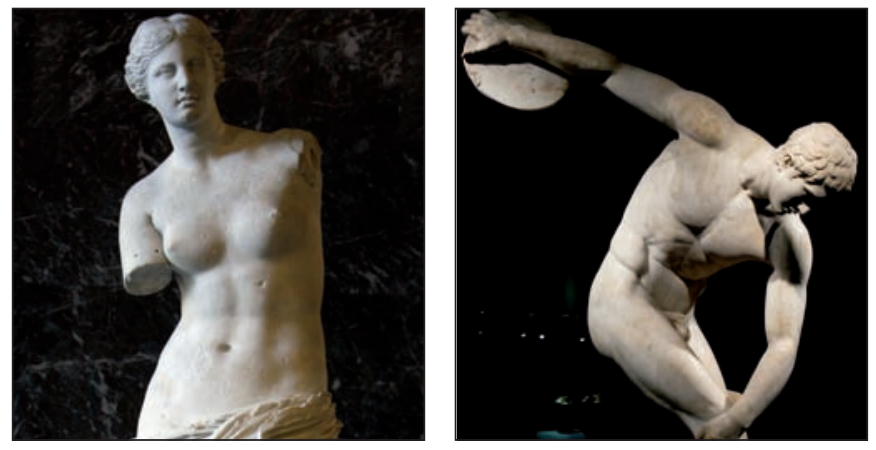

Fig. 1. A. Venus de Milo de Alexandros de Antioquía, esculpida entre el 130 y 100 AC. B. El Discóbolo, por Mirón de Eléuteras, 450 AC. En ambas figuras se
representa una forma atlética, un cuerpo magro y de musculatura definida.

\section{Material y método}

Realizamos un estudio descriptivo retrospectivo, mediante revisión de tomografías computarizadas de abdomen y pelvis en pacientes del Hospital Clínico de la Universidad de Chile (Santiago, Chile), obtenidos de la base de imágenes de nuestro centro entre enero y abril de 2017. Tomamos una muestra aleatoria de 200 pacientes estratificados por sexo, que cumplieran con los siguientes criterios de inclusión: mayores de 18 y menores de 65 años de edad, con estudio tomográfico de abdomen y pelvis con secuencia coronal. Excluimos a los pacientes que presentaban las siguientes características: patología de la pared abdominal, cirugía abdominal previa o estudio imagenológico inadecuado para evaluación de pared abdominal. Obtuvimos todos los datos clínicos de la ficha, en forma anónima.

A todos los pacientes se les realizó una tomografía computarizada (TC) de abdomen y pelvis bajo protocolo sin contraste o pieloTC. El equipo empleado fue Siemens ${ }^{\circledR}$ Somaton Definition Edge (Siemens Healthcare Headquarters, Alemania), analizando la conformación metamérica del músculo recto abdominal en secuencia coronal (Fig. 3).

Obtuvimos los siguientes datos: sexo; número de inserciones tendinosas supraumbilicales e infraumbilicales (incompletas); ${ }^{(5)}$ ángulo costal corto, formado entre ambos rebordes de los cartílagos costo-esternales con el xifoides; ángulo costal completo, formado entre los puntos más laterales del reborde costal y el xifoides; diámetro xifo-umbilical; y diámetro umbílico-púbico.

$\mathrm{El}$ análisis estadístico empleado fue descriptivo, utilizando medidas de tendencia central y dispersión. Para determinar la relación entre las mediciones realizamos análisis de la varianza, análisis de tendencias y análisis de regresión lineal. Todo mediante programa STATA ${ }^{\circledR}$ v13 (StataCorp, EE.UU.) considerando estadísticamente significativo un valor $\mathrm{p}<0.05$; calculamos todos los intervalos de confianza al $95 \%$.

\section{RESULTADOS}

\section{Descripción general de los pacientes}

Analizamos 200 pacientes, de los cuales 100 (50\%) fueron varones. La media de edad de los pacientes analizados fue de $40.9 \pm 1.1$ años (rango de 18 a 65 años). Cuarenta y dos pacientes (21\%) tenían 2 metámeros, 154 (77\%) 3 metámeros y 4 (2\%) 4 metámeros. Observamos inserciones tendinosas incompletas en 25 pacientes (12.5\%).

Con una distancia xifo-umbilical promedio de 202.35 $\pm 28.21 \mathrm{~mm}$ (rango de 109 a $273 \mathrm{~mm}$ ) y umbílico-púbica de $167.07 \pm 17.82 \mathrm{~mm}$ (rango de 92 a $233 \mathrm{~mm}$ ), el ángulo costo-xifoideo fue de $99.24 \pm 14.47^{\circ}$ y el ángulo condroxifoideo de $74.79 \pm 15.6^{\circ}$.

Al realizar el análisis por sexos, no evidenciamos diferencias significativas tanto en la distribución del número de metámeros $(\mathrm{p}=0.201)$, el número de metámeros 

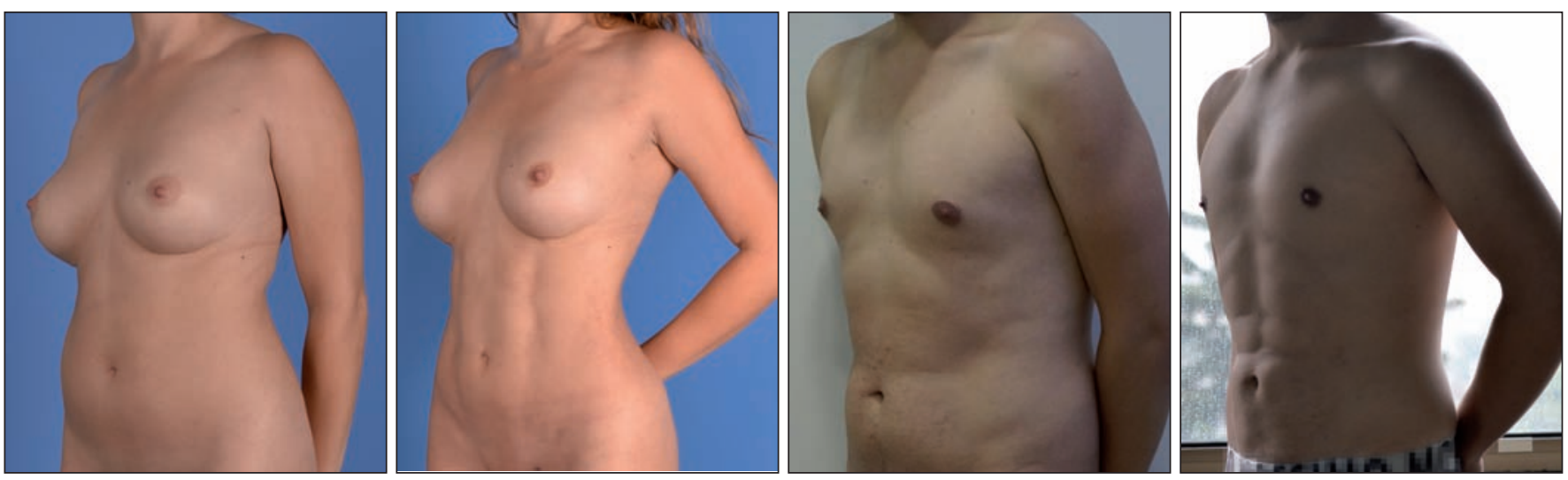

Fig. 2. Pre y postoperatorio a los 3 meses de pacientes masculino y femenino tratados mediante lipoescultura de alta definición.
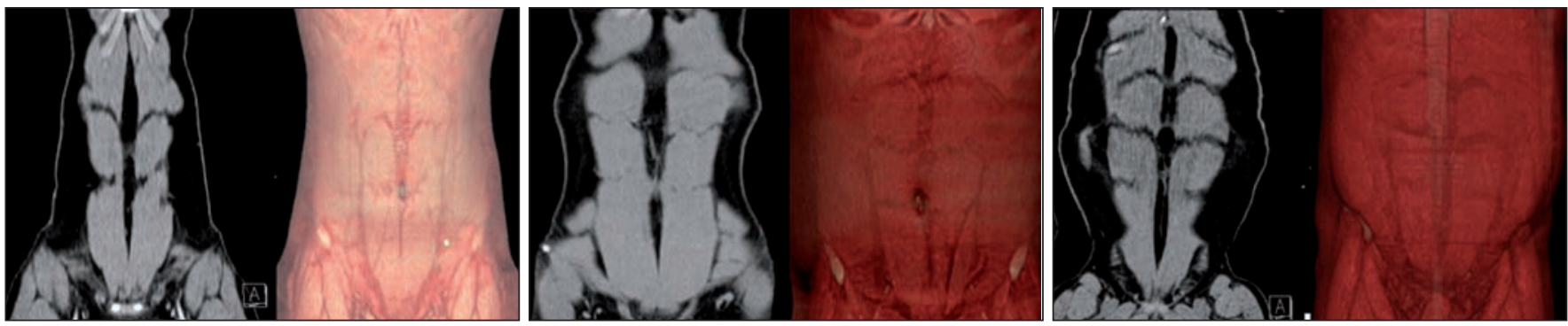

Fig. 3. Tomografía computarizada de abdomen y pelvis sin contraste, corte coronal curvo y reconstrucción 3D de pared abdominal. Observamos los tres patrones metaméricos, sobre el nivel del ombligo, de la pared abdominal A. Dos metámeros. B. Tres metámeros. C. Cuatro metámeros.

incompletos bajo el nivel del ombligo $(\mathrm{p}=0.393)$, distancias xifo-umbilical ( $\mathrm{p}=0.185)$ y umbílico-púbica $(0.327)$.

En relación a la conformación de la parrilla costal observamos una mayor angulación en los pacientes de sexo femenino: ángulo condro-xifoideo $69.4 \pm 15.2$ frente a $80.2 \pm 14.2, \mathrm{p}<0.001$ y ángulo costo-xifoideo de 96.71 $\pm 14.70^{\circ}$ frente a $101.79 \pm 13.82^{\circ}, \mathrm{p}=0.012$. (Tabla I).

\section{Relaciones del número de metámeros y otras medidas antropométricas}

Al comparar el número de metámeros con la ubicación relativa del ombligo, observamos que los pacientes con mayor número de metámeros presentan una menor distancia umbílico-púbica ( $\mathrm{p}=0.046$ ) (Tabla II).

El número de metámeros presentes en el músculo recto abdominal de un paciente presenta una relación inversa con el tamaño de los metámeros (longitud promedio entre metámeros $)(\mathrm{p}=0.001$ para primer metámero, $\mathrm{p}=0.005$ para segundo metámero $\mathrm{y} \mathrm{p}=0.021$ para tercer metámero) (Tabla III).

No observamos relación entre el grado de angulación del reborde costo-condral y el número de metámeros del músculo recto abdominal, ángulo condro-xifoideo $\mathrm{p}=0.858$ y ángulo costo-xifoideo $\mathrm{p}=0.275$ (Tabla III).

\section{Discusión}

Los resultados de nuestro estudio muestran que la anatomía más frecuente del músculo recto abdominal presenta 3 metámeros sobre el ombligo, 6-pack con una prevalencia del $77 \%$, seguida por la presencia de 2 me- támeros, 4-pack, y 4 metámeros, 8-pack, con frecuencias del $21 \%$ y del $2 \%$ respectivamente. No existen datos a nivel nacional sobre la anatomía habitual de este músculo; a nivel internacional, si bien la anatomía más frecuente parece corresponder a la de 3 metámeros, esta presenta una prevalencia que fluctúa entre el 61 y el $97 \%,{ }^{(9,11)}$ lo cual concuerda con nuestro estudio.

Existe gran dispersión sobre la prevalencia de las diferentes variantes anatómicas. Meenakshi ${ }^{(11)}$ describe en disección cadavérica un $14.6 \%$ de 2 inserciones y un $21.95 \%$ para 4 inserciones, mientras Anita y col. ${ }^{\left({ }^{8}\right)}$ describen un $1.85 \%$ para 4 y 5 inserciones tendinosas. Existen incluso reportes de casos de disecciones cadavéricas de músculos rectos sin inserciones tendinosas. ${ }^{(4)}$

Hasta donde hemos podido comprobar en las bases de datos Pubmed y LILACS, no hemos encontrado estudios que permitan predecir la configuración anatómica de un paciente basada en variables antropométricas. Al analizar nuestros resultados, es posible plantear que las distintas variantes anatómicas observadas no presentan asociación con el sexo, con la longitud total del abdomen o con el grado de angulación del reborde costal, siendo la ubicación relativa del ombligo entre el xifoides y el pubis la única asociación que presenta una significancia estadística significativa. Los sujetos con un ombligo más bajo, es decir, con menor distancia umbílico-púbica relativa, presentan mayor probabilidad de tener menor número de metámeros.

Consideramos que la principal debilidad de nuestro estudio es el no poder determinar la relación entre el número y el tamaño de los metámeros del músculo recto 
Danilla-Enei, S., Domínguez-Contreras, C., Cuneo-Barbosa, N., López-García, L., León-Cabrera, E., Cisternas-Vera, J.P., Erazo-Cortés, C., Andrades-Cvitanic P., Sepúlveda-Pereira, S.

Tabla I. Características de los pacientes evaluados en nuestro grupo de estudio

\begin{tabular}{|c|c|c|c|c|}
\hline Variable & General & Masculino & Femenino & Valor $\mathbf{p}$ \\
\hline Edad & $40.99 \pm 12.13$ & $41.63 \pm 11.86$ & $40.35 \pm 12.42$ & 0.457 \\
\hline Sexo & 200 & $100(50 \%)$ & $100(50 \%)$ & \\
\hline $\begin{array}{l}\text { Numero de metámeros } \\
2 \\
3 \\
4\end{array}$ & $\begin{array}{c}42(21 \%) \\
154(77 \%) \\
4(2)\end{array}$ & $\begin{array}{c}26(26 \%) \\
73(73 \%) \\
1(1 \%)\end{array}$ & $\begin{array}{c}16(16 \%) \\
81(81 \%) \\
3(3 \%)\end{array}$ & 0.201 \\
\hline Inserciones incompletas & $25(12.5 \%)$ & $15(15 \%)$ & $10(10 \%)$ & 0.393 \\
\hline Distancia xifo-umbilical (mm) & $202.35 \pm 28.21$ & $204.98 \pm 29.28$ & $199.69 \pm 26.98$ & 0.185 \\
\hline Distancia umbílico-púbica (mm) & $167.07 \pm 17.82$ & $168.85 \pm 16,47$ & $165.29 \pm 18.99$ & 0.327 \\
\hline Ángulo costo-xifoideo & $99.25 \pm 14.46^{\circ}$ & $101.79 \pm 13.82$ & $96.71 \pm 14.70^{\circ}$ & 0.012 \\
\hline Ángulo condro-xifoideo & $74.79 \pm 12.13^{\circ}$ & $80.19 \pm 14.19$ & $69.40 \pm 15.20^{\circ}$ & 0.001 \\
\hline
\end{tabular}

Tabla II. Medición de distancias según número de metámeros presentes en los pacientes de nuestro grupo de estudio

\begin{tabular}{|c|c|c|c|c|}
\hline \multirow[b]{2}{*}{ Distancias (cm) } & \multicolumn{3}{|c|}{ Longitud promedio } & \multirow{2}{*}{ Valor $\mathbf{p}$} \\
\hline & $\begin{array}{c}2 \text { metámeros } \\
\text { (4-pack) }\end{array}$ & $\begin{array}{c}3 \text { metámeros } \\
\text { (6-pack) }\end{array}$ & $\begin{array}{c}4 \text { metámeros } \\
\text { (8-pack) }\end{array}$ & \\
\hline Xifo-umbilical & $19.9 \pm 2.9$ & $20.3 \pm 2.8$ & $21.3 \pm 2.8$ & 0.969 \\
\hline Umbílico-púbico & $17.4 \pm 1.8$ & $16.5 \pm 1.8$ & $16 \pm 1.8$ & 0.046 \\
\hline Xifo-púbica & $37.3 \pm 3.6$ & $36.8 \pm 3.1$ & $37.3 \pm 2.8$ & 0.544 \\
\hline Relacion $\mathrm{x}-\mathrm{u} / \mathrm{u}-\mathrm{p}$ & $1.16 \pm 0.2$ & $1.24 \pm 0.25$ & $1.34 \pm 0.27$ & 0.044 \\
\hline
\end{tabular}

Tabla III. Medición de ángulos según patrón conformacional del músculo recto abdominal en los pacientes de nuestro grupo de estudio

\begin{tabular}{|c|c|c|c|c|}
\hline \multirow[b]{2}{*}{ Ángulo } & \multicolumn{3}{|c|}{ Angulación } & \multirow{2}{*}{ Valor $p$} \\
\hline & $\begin{array}{c}2 \text { metámeros } \\
\text { (4-pack) }\end{array}$ & $\begin{array}{c}3 \text { metámeros } \\
\text { (6-pack) }\end{array}$ & $\begin{array}{c}4 \text { metámeros } \\
\text { (8-pack) }\end{array}$ & \\
\hline Costal corto & $73.9 \pm 15.4^{\circ}$ & $74.9 \pm 15.7^{\circ}$ & $78 \pm 19.3$ & 0.858 \\
\hline Costal largo & $97.3 \pm 17.6^{\circ}$ & $99.7 \pm 13.3^{\circ}$ & $100.3 \pm 14.6$ & 0.275 \\
\hline
\end{tabular}

abdominal y la talla de los pacientes; esto se debió a que esta variable no queda registrada en los estudios tomográficos de nuestro Servicio de Imagenología.

En base a los resultados presentados, el cirujano plástico deberá tener en cuenta en la planificación preoperatoria de procedimientos tales como lipoescultura de alta definición ${ }^{(4)}$ y lipoinyección grasa del músculo recto abdominal (Rectus Abdominis Fat Transfer- RAFT) en pacientes de cirugía de contorno corporal, ${ }^{(12)}$ que la mayoría de los pacientes tendrán 3 metámeros sobre el ombligo y que si se trata de un paciente con ombligo alto, y no logra palpar los 3 metámeros por lado, es posible que solo tenga 2.

\section{Conclusiones}

En nuestro estudio, realizado sobre población chilena, la anatomía más frecuente del músculo recto abdominal corresponde a 3 metámeros supraumbilicales por cada recto abdominal. El número de metámeros no depende del sexo, del ángulo costal o de la distancia xifo-pubiana. Cuanto más alto esté el ombligo en relación a la distancia xifo-pubiana, menos metámeros tiene el recto abdominal. En los pacientes estudiados no fue posible predecir de forma precisa el número de metámeros mediante otra medida antropométrica. 


\section{Dirección del autor}

Dr. Stefan Danilla Enei

Departamento de Cirugía

Hospital Clínico Universidad de Chile

Santos Dumont 999, Independencia

Santiago, Chile

Correo electrónico: drstefandanilla@gmail.com

\section{Bibliografía}

1. Standring S. Anterior Abdominal wall, Gray's Anatomy The Anatomical Basis of clinical practice, Spain, Elsevier Limited, 40th Edition,2008, Pp:1055-1068.

2. Monkhouse W, Khalique A. Variations in the composition of the human rectus sheath: a study of the anterior abdominal wall. J. of Anatomy, 2017,13;145:61-66.

3. Yang JD, Hwang HP, Kim JH, Rodríguez-Vázquez JF, Abe S-I, Murakami G, et al. Development of the Rectus Abdominis and Its Sheath in the Human Fetus. Yonsei Med J. 2012;53(5):1028-1028.

4. Aktan Kiz ZA. Bilateral absence of the tendinous intersections of the rectus abdominis muscle. Anatomy, 2009;3:69-71.
5. Punekar IRA, Khouri JS, Catanzaro M, Shaikh AL, Langstein HN. Redefining the Rectus Sheath: implications for Abdominal Wall Repair. Plast. Reconst. Surg. 2018; 141(2): 473-479.

6. Mentz HA, Gilliland MD, Patronella CK. Abdominal etching: differential liposuction to detail abdominal musculature. Aesthet. Plast Surg. 1993;17(4):287-290.

7. Hoyos A, Millard J. VASER-assisted high-definition liposculpture. Aesthet Surg J. 2007;27(6):594-604.

8. Anita G, Sinha M, Sinha S. Anatomical Studies with Clinical Importance of Unusual Patterns of Abdominal Muscles in North Indian Population. Int. J. of Current Medical And Applied Sciences. 2015; 21;7(2):86-90.

9. Anita G, Haque M, Gupta A, Nasar A. Variation in Tendinous Intersections of Rectus Abdominis Muscle in North Indian Population with Clinical Implications. JCDR; 2015; 9(6): 10-12.

10. Mwachaka P, Odula P, Awori K, Kaisha W. Variations in the Pattern of Formation of the Abdominis Rectus Muscle Sheath among Kenyans. Int J Morphol. 2009;27(4):1025-1029.

11. Meenakshi S, Manjunath KY. The tendinous intersections of rectus abdominis muscle. J. of Mahatma Gandhi Institute of Medical Sciences. 2008;13(1):34-39.

12. Danilla S. Rectus Abdominis Fat Transfer (RAFT) in Lipoabdominoplasty: A New Technique to Achieve Fitness Body Contour in Patients that Require Tummy Tuck. Aesthe. Plast Surg. 2017;12:1-11. 